\title{
Enumeration of unlabeled directed hypergraphs
}

\author{
Jianguo Qian* \\ School of Mathematical Sciences, Xiamen University \\ Xiamen 361005, Fujian, P.R. China \\ jgqian@xmu.edu.cn
}

Submitted: Oct 4, 2012; Accepted: Feb 21, 2013; Published: Mar 1, 2013

Mathematics Subject Classifications: 05C30, 05C65, 05C20

\begin{abstract}
We consider the enumeration of unlabeled directed hypergraphs by using Pólya's counting theory and Burnside's counting lemma. Instead of characterizing the cycle index of the permutation group acting on the hyperarc set $A$, we treat each cycle in the disjoint cycle decomposition of a permutation $\rho$ acting on $A$ as an equivalence class (or orbit) of $A$ under the operation of the group generated by $\rho$. Compared to the cycle index method, our approach is more effective in dealing with the enumeration of directed hypergraphs. We deduce the explicit counting formulae for the unlabeled $q$-uniform and unlabeled general directed hypergraphs. The former generalizes the well known result for 2-uniform directed hypergraphs, i.e., for the ordinary directed graphs introduced by Harary and Palmer.
\end{abstract}

Keywords: unlabeled directed hypergraph; uniform; enumeration

\section{Introduction}

In 1937, Pólya [16] developed a powerful theory for treating the symmetries of graphs or more in general, certain types of discrete configurations under a given permutation group, which nowadays is known as Pólya's theorem or Redfield-Pólya theorem and represents one of the cornerstones of modern combinatorics. Theoretically, this theory provides us with a universal technique to count the unlabeled graphs or in particular, the graphs which meet some specific requirements. Following Pólya, the enumeration problem for various types of graphs were studied in the literature. For examples, some results on ordinary graphs and hypergraphs can be found in $[3,5,7,8,9,10,11,14,17]$ and $[4,12,15,18,19,21]$, respectively. In particular, the pre-1973 work on this problem was nicely included in the text book [9] written by Harary and Palmer.

${ }^{*}$ Supported by NSFC grant No. 10831001. 
In this paper, we consider the enumeration of unlabeled directed hypergraphs, i.e., non-isomorphic directed hypergraphs. A directed hypergraph $D$ is a pair $\langle V, A\rangle$, where $V$ is the vertex set and $A$ is its hyperarc set. As a generalization of directed graph, a hyperarc $a$ or simply, an arc $a$, in a directed hypergraph is a nonempty vertex subset with a specified vertex called its source or root. That is, $a$ is an ordered pair $\langle v, W\rangle$ with $v \in V$ and $W \subseteq V \backslash\{v\}$. The set $W$ is also called the sink set of the arc [1,6, 13, 20]. A directed hypergraph $D=\langle V, A\rangle$ is called q-uniform $(1 \leqslant q \leqslant|V|)$ if each arc $\langle v, W\rangle$ consists of exactly $q$ vertices (including its root), i.e., $|W|=q-1$. Generally, a directed hypergraph in which the sink set $W$ can consist of any number of vertices is called a general directed hypergraph. In addition, all the hypergraphs considered here are simple, i.e., the multiple arcs are not allowed.

We apply Pólya's counting theory and Burnside's counting lemma to our enumeration problem. In general, the key point of Pólya's theory to treat the number of cycles in the disjoint cycle decomposition of the permutations is to characterize the cycle index of the involved permutation group, which has been widely used in graphical enumeration. However, to characterize the cycle index may become particularly complex for some cases, e.g., for undirected hypergraph $[12,18,21]$, the standard and frequently used method for which is to use the generating function.

We will, however, not try to characterize the cycle index. Instead, we consider the number of cycles from a different point of view which arises from a simple observation, i.e.: a cycle in a permutation $\pi$ acting on a set $X$ could be regarded as an equivalence class (or orbit) of $X$ under the operation of the group generated by $\pi$.

Compared to the cycle index method based on generating function, our approach is more effective in dealing with the enumeration of directed hypergraphs. We deduce the explicit counting formulae for the unlabeled $q$-uniform directed hypergraphs with $1 \leqslant q \leqslant|V|$ and unlabeled general directed hypergraphs. The former generalizes the well known result for the ordinary directed graphs introduced by Harary and Palmer [9]. Some numerical results are also listed, as examples.

\section{Main results}

Let $\rho$ be a permutation acting on a set $X$ and let $\Omega(\rho)$ be the number of cycles in the disjoint cycle decomposition of $\rho$. As mentioned in the previous section, $\Omega(\rho)$ could be regarded as the number of equivalence classes (or orbits) of $X$ under the operation of the group generated by $\rho$ : i.e., $\langle\rho\rangle=\left\{\rho, \rho^{2}, \cdots, \rho^{|\rho|}\right\}$, where $|\rho|$ is the order of $\rho$. Thus, by Burnside's lemma [2] we get the following observation.

Observation 1. The number of cycles in the disjoint cycle decomposition of a permutation $\rho$ is

$$
\Omega(\rho)=\frac{1}{|\rho|} \sum_{i=1}^{|\rho|} \Psi\left(\rho^{i}\right),
$$

where $\Psi\left(\rho^{i}\right)$ is the number of elements of $X$ left fixed by $\rho^{i}$, i.e., invariant under $\rho^{i}$. 
We now turn to the enumeration problem of the directed hypergraphs. In the following, the vertex set of a directed hypergraph $D$ of order $n$ is always set to be $[n]=\{1,2, \cdots, n\}$. In terms of the Pólya's counting theory, we model a directed hypergraph $D$ (general or uniform) as an arc coloring of the complete directed hypergraph $K_{n}$ (general or uniform) of order $n$ using two colors. Thus, the problem is equivalent to determining the number of equivalence coloring classes of $K_{n}$ under the operation of the group induced by the automorphism group $S_{n}$ of $K_{n}$, i.e., the symmetry group on $[n]$.

For a partition $P$ of $n$, we will write it either as the form $P: p_{1}+p_{2}+\cdots+p_{k}$ or as $P: 1 \alpha_{1}+2 \alpha_{2}+\cdots+n \alpha_{n}$, for the convenience of our discussion, where $\alpha_{i}$ is the number of the integers $i$ in the partition. Given natural numbers $a_{1}, a_{2}, \cdots, a_{m}$, we denote by $\left(a_{1}, a_{2}, \cdots, a_{m}\right)$ and $\left[a_{1}, a_{2}, \cdots, a_{m}\right]$ the greatest common divisor and the least common multiple of $a_{1}, a_{2}, \cdots, a_{m}$, respectively.

A permutation $\pi \in S_{n}$ with the disjoint cycle decomposition $\pi=\sigma_{1} \sigma_{2} \cdots \sigma_{k}$ induces a partition of $n$, i.e., $P: p_{1}+p_{2}+\cdots+p_{k}$, where $p_{i}$ is the length of the cycle $\sigma_{i}$. Conversely, it is well known [9] that a partition $P: 1 \alpha_{1}+2 \alpha_{2}+\cdots+n \alpha_{n}$ of $n$ induces

$$
\frac{n !}{1^{\alpha_{1}} 2^{\alpha_{2}} \cdots n^{\alpha_{n}} \alpha_{1} ! \alpha_{2} ! \cdots \alpha_{n} !}
$$

permutations in $S_{n}$, each with disjoint cycle decomposition of the form $1^{\alpha_{1}} 2^{\alpha_{2}} \cdots n^{\alpha_{n}}$, where $i^{\alpha_{i}}$ represents the product of $\alpha_{i}$ cycles of length $i, i=1,2, \cdots, n$. For a permutation $\pi \in S_{n}$, we denote by $\rho(\pi)$ the permutation acting on the arc set induced by $\pi$. For simplicity, we also use $\rho(P)$ to denote $\rho(\pi(P))$, where $\pi(P)$ is an arbitrary permutation in $S_{n}$ induced by $P$.

Let $\mathcal{P}(n)$ be the class of all the partitions of $n$. Then by Burnside's lemma [2], the number of unlabeled directed hypergraphs of order $n$ is given by

$$
d(n)=\frac{1}{n !} \sum_{\pi \in S_{n}} \Phi(\rho(\pi))=\frac{1}{n !} \sum_{P \in \mathcal{P}(n)} \frac{n !}{1^{\alpha_{1}} 2^{\alpha_{2}} \cdots n^{\alpha_{n}} \alpha_{1} ! \alpha_{2} ! \cdots \alpha_{n} !} \Phi(\rho(P)),
$$

where $\Phi(\rho(P))$ (resp., $\Phi(\rho(\pi)))$ is the number of colorings left fixed by $\rho(P)$ (resp., by $\rho(\pi))$. In terms of the cycle index, $\Phi(\rho(P))$ can be represented as $2^{\Omega(\rho(P))}$, where $\Omega(\rho(P))$ is the number of cycles in $\rho(P)$. We notice that the order of the permutation $\rho(P)$ is $\left[p_{1}, p_{2}, \cdots, p_{k}\right]$. Thus, by Observation 1 ,

$$
d(n)=\sum_{P \in \mathcal{P}(n)} \frac{1}{1^{\alpha_{1}} 2^{\alpha_{2}} \cdots n^{\alpha_{n}} \alpha_{1} ! \alpha_{2} ! \cdots \alpha_{n} !} 2^{\Omega(\rho(P))},
$$

where

$$
\Omega(\rho(P))=\frac{1}{\left[p_{1}, p_{2}, \cdots, p_{k}\right]} \sum_{t=1}^{\left[p_{1}, p_{2}, \cdots, p_{k}\right]} \Psi\left(\rho^{t}(P)\right),
$$

and $\Psi\left(\rho^{t}(P)\right)$ is the number of arcs left fixed by $\rho^{t}(P)$.

Let $\pi$ be induced by a partition $P: p_{1}+p_{2}+\cdots+p_{k}$ having the disjoint cycle decomposition

$$
\pi=\left(n_{11} n_{12} \cdots n_{1 p_{1}}\right)\left(n_{21} n_{22} \cdots n_{2 p_{2}}\right) \cdots\left(n_{k 1} n_{k 2} \cdots n_{k p_{k}}\right) .
$$


Let $\left\langle u_{r s}, Q\right\rangle$ be an arc of a directed hypergraph, where

$$
Q=Q_{1} \cup Q_{2} \cup \cdots \cup Q_{k}, Q_{i} \subseteq\left\{n_{i 1}, n_{i 2}, \cdots, n_{i p_{i}}\right\}, i=1,2, \cdots, k,
$$

and $u_{r s} \in\left\{n_{r 1}, n_{r 2}, \cdots, n_{r p_{r}}\right\} \backslash Q_{r}$. We call $r$ the root subscript of $\left\langle u_{r s}, Q\right\rangle$. It is clear that the arc $\left\langle u_{r s}, Q\right\rangle$ restricted on $\left\{n_{r 1}, n_{r 2}, \cdots, n_{r p_{r}}\right\}$ is $\left\langle u_{r s}, Q_{r}\right\rangle$.

By the definition of the directed hypergraphs, the arc $\left\langle u_{r s}, Q\right\rangle$ is left fixed by $\rho\left(\pi^{t}\right)$ if and only if $u_{r s}$ is left fixed by $\pi^{t}$ and $Q$ is left fixed by $\rho\left(\pi^{t}\right)$, i.e., $\pi^{t}\left(u_{r s}\right)=u_{r s}$ and $\rho\left(\pi^{t}\right)(Q)=Q$. Similarly, $\left\langle u_{r s}, Q_{r}\right\rangle$ is left fixed by $\rho\left(\pi^{t}\right)$ if and only if $\pi^{t}\left(u_{r s}\right)=u_{r s}$ and $\rho\left(\pi^{t}\right)\left(Q_{r}\right)=Q_{r}$.

For a subset $Q_{i}$, we write it as a $(0,1)$-sequence: $Q_{i}=a_{i 1} a_{i 2} \cdots a_{i p_{i}}$, where $a_{i j}=1$ if $n_{i j} \in Q_{i}$ and $a_{i j}=0$ for otherwise. One can see that $Q_{i}$ is left fixed by $\rho(\pi)$ if and only if $a_{i j}=a_{i, j+1\left(\bmod p_{i}\right)}$ for any $j \in\left\{1,2, \cdots, p_{i}\right\}$. In general, we have the following result.

Lemma 2. Let $i \in\{1,2, \cdots, k\}$ and $t$ be a positive integer.

1). If $i \neq r$ then $Q_{i}$ is left fixed by $\rho\left(\pi^{t}\right)$ if and only if

$$
a_{i j}=a_{i, j+\eta\left(\bmod p_{i}\right)}
$$

for any $j \in\left\{1,2, \cdots, p_{i}\right\}$, where $\eta=\left(t, p_{i}\right)$;

2). $\left\langle u_{r s}, Q_{r}\right\rangle$ is left fixed by $\rho\left(\pi^{t}\right)$ if and only if $t$ is a multiple of $p_{r}$.

Proof. 1). Notice that $\pi^{t}\left(n_{i j}\right)=n_{i, j+t\left(\bmod p_{i}\right)}=n_{i, j+\eta h\left(\bmod p_{i}\right)}$, where $h=t / \eta$. Therefore, the condition $a_{i j}=a_{i, j+\eta\left(\bmod p_{i}\right)}$ implies that $n_{i j} \in Q_{i}$ if and only if $\pi^{t}\left(n_{i j}\right) \in Q_{i}$, i.e., $Q_{i}$ is left fixed by $\rho\left(\pi^{t}\right)$. The sufficiency now follows.

Conversely, assume that $Q_{i}$ is left fixed by $\rho\left(\pi^{t}\right)$, i.e., $\rho\left(\pi^{t}\right)\left(Q_{i}\right)=Q_{i}$. Then $\pi^{t}$ restricted on $Q_{i}$ is a permutation, i.e., $n_{i j} \in Q_{i}$ if and only if $\pi^{t}\left(n_{i j}\right) \in Q_{i}$. On the other hand, again notice that $\pi^{t}\left(n_{i j}\right)=n_{i, j+t\left(\bmod p_{i}\right)}$. Hence, $n_{i j} \in Q_{i}$ if and only if $n_{i, j+t\left(\bmod p_{i}\right)} \in Q_{i}$ and therefore, $n_{i, j+m t\left(\bmod p_{i}\right)} \in Q_{i}$ for any integer $m$. Now since $p_{i} / \eta$ and $t / \eta$ are relatively prime, the equation

$$
\frac{p_{i}}{\eta}+1 \equiv m \frac{t}{\eta}\left(\bmod p_{i}\right)
$$

has an integer solution $m$ with $1 \leqslant m<\left(p_{i} / \eta\right)(t / \eta)$. Thus, we have $l p_{i}+\eta=m t$, i.e., $\eta \equiv m t\left(\bmod p_{i}\right)$, where $l$ is an integer. This implies that $j+m t=j+\eta\left(\bmod p_{i}\right)$. Equivalently, $n_{i j} \in Q_{i}$ if and only if $n_{i, j+\eta\left(\bmod p_{i}\right)} \in Q_{i}$, i.e., $a_{i j}=a_{i, j+\eta(\bmod ) p_{i}}$ for each $j \in\left\{1,2, \cdots p_{i}\right\}$.

2). We notice that if $t$ is a multiple of $p_{r}$, then $Q_{r}$ is left fixed by $\rho\left(\pi^{t}\right)$. Therefore, 2) follows directly since $u_{r s}$ is left fixed by $\pi^{t}$ if and only if $t$ is a multiple of $p_{r}$. This completes our proof.

\subsection{General directed hypergraphs}

By the definition of the directed hypergraphs, we note that any arc consists of at least one vertex, i.e., the root vertex. 
Table 1: Numerical results for $d^{*}(n)$ with $n=1,2, \cdots, 12$.

\begin{tabular}{cr}
\hline$n$ & $d^{*}(n)$ \\
\hline 1 & 2 \\
2 & 10 \\
3 & 752 \\
4 & 179228736 \\
5 & 10074382205972351614976 \\
6 & $87181968547037232901944803346094 \times 10^{23}$ \\
7 & $14421403259833470050581821585079 \times 10^{100}$ \\
8 & $44585643225751882632175227946156 \times 10^{272}$ \\
9 & $10312096701091637545105819481752 \times 10^{657}$ \\
10 & $51738611172921010385604860571960 \times 10^{1503}$ \\
11 & $15875062400125167572370589504655 \times 10^{3352}$ \\
12 & $27091713481721153886294658285718 \times 10^{7358}$ \\
\hline
\end{tabular}

By Lemma 2, if $Q_{i}(i \neq r)$ is left fixed by $\rho\left(\pi^{t}\right)$ then the sequence $Q_{i}=a_{i 1} a_{i 2} \cdots a_{i p_{i}}$ is determined uniquely by its subsequence $a_{i 1} a_{i 2} \cdots a_{i \eta}$ since $\eta$ divides $p_{i}$. The number of such subsequences, i.e., the $(0,1)$-sequences of length $\eta$, is clearly $2^{\eta}$. Again by Lemma 2 , $\left\langle u_{r s}, Q_{r}\right\rangle$ is left fixed by $\rho\left(\pi^{t}\right)$ if and only if $t$ is a multiple of $p_{r}$, i.e., $t=m p_{r}$ for some integer $m$. The number of such pairs $\left\langle u_{r s}, Q_{r}\right\rangle$ is clearly $2^{p_{r}-1} p_{r}$.

On the other hand, an arc $\left\langle u_{r s}, Q\right\rangle$ is left fixed by $\rho\left(\pi^{t}\right)$ if and only if $\left\langle u_{r s}, Q_{r}\right\rangle$ and $Q_{i}$ are left fixed by $\rho\left(\pi^{t}\right)$ for each $i \in\{1,2, \cdots, k\} \backslash\{r\}$. In this case we must have $t=m p_{r}$. Therefore, the number of such arcs, i.e., $\Psi^{*}\left(\rho^{t}(P)\right)$, is exactly

$$
2^{p_{r}-1} p_{r} \prod_{i \neq r} 2^{\left(m p_{r}, p_{i}\right)}
$$

Thus, combining with (2) and (3) we reach the following result immediately.

Theorem 3. Let $d^{*}(n)$ denote the number of unlabeled general directed hypergraphs of order $n$ and let

$$
\Omega^{*}(P)=\frac{1}{\left[p_{1}, p_{2}, \cdots, p_{k}\right]} \sum_{r=1}^{k} 2^{p_{r}-1} p_{r} \sum_{m=1}^{\left[p_{1}, p_{2}, \cdots, p_{k}\right] / p_{r}} \prod_{i \neq r} 2^{\left(m p_{r}, p_{i}\right)} .
$$

Then

$$
d^{*}(n)=\sum_{P \in \mathcal{P}(n)} \frac{1}{1^{\alpha_{1}} 2^{\alpha_{2}} \cdots n^{\alpha_{n}} \alpha_{1} ! \alpha_{2} ! \cdots \alpha_{n} !} 2^{\Omega^{*}(P)} .
$$

By Theorem 3, the numerical results for the number of unlabeled general directed hypergraphs with the number of vertices from 1 up to 12 are listed in Table 1, as an example. 
Example 4. The 10 non-isomorphic directed hypergraphs of order 2 are listed as follows (for simplicity, we only list their arc sets):

$$
\begin{array}{lll}
\{\langle 1, \emptyset\rangle,\langle 2, \emptyset\rangle\}, & \{\langle 1,\{2\}\rangle,\langle 2,\{1\}\rangle\}, & \{\langle 1,\{2\}\rangle,\langle 1, \emptyset\rangle,\langle 2,\{1\}\rangle,\langle 2, \emptyset\rangle\}, \\
\{\langle 1,\{2\}\rangle\}, & \{\langle 1,\{2\}\rangle,\langle 1, \emptyset\rangle\}, & \{\langle 1,\{2\}\rangle,\langle 1, \emptyset\rangle,\langle 2,\{1\}\rangle\}, \\
\{\langle 1, \emptyset\rangle\}, & \{\langle 1,\{2\}\rangle,\langle 2, \emptyset\rangle\}, & \{\langle 1,\{2\}\rangle,\langle 1, \emptyset\rangle,\langle 2, \emptyset\rangle\},
\end{array}
$$

$\emptyset$.

\section{$2.2 q$-uniform directed hypergraphs}

Given $k$ non-negative integers $q_{1}, q_{2}, \cdots, q_{k}$ with $q_{1}+q_{2}+\cdots+q_{k}=q-1$, denote

$$
\begin{gathered}
\mathcal{Q}=\mathcal{Q}\left(q_{1}, q_{2}, \cdots, q_{k}\right) \\
=\left\{Q=Q_{1} \cup Q_{2} \cup \cdots \cup Q_{k}:\left|Q_{i} \cap\left\{n_{i 1}, n_{i 2}, \cdots, n_{i p_{i}}\right\}\right|=q_{i}, i=1,2, \cdots, k\right\} .
\end{gathered}
$$

Let $\xi=q_{i} \eta / p_{i}$ if $p_{i} / \eta$ divides $q_{i}$. By Lemma 2, if $Q_{i}$ is left fixed by $\rho\left(\pi^{t}\right)$ then the sequence $Q_{i}=a_{i 1} a_{i 2} \cdots a_{i p_{i}}$ is determined uniquely by its subsequence $a_{i 1} a_{i 2} \cdots a_{i \eta}$. In this case, if such $Q_{i}$ exists then $p_{i} / \eta$ must divide $q_{i}$ and consequently, the subsequence $a_{i 1} a_{i 2} \cdots a_{i \eta}$ consists of exactly $q_{i} /\left(p_{i} / \eta\right)=q_{i} \eta / p_{i}=\xi$ elements 1 . The number of such subsequences, i.e., the (0,1)-sequences of length $\eta$ consisting of exactly $\xi$ elements 1 , is clearly $\left(\begin{array}{l}\eta \\ \xi\end{array}\right)$ if $p_{i} / \eta$ divides $q_{i}$ and is zero for otherwise. Again by Lemma $2,\left\langle u_{r s}, Q_{r}\right\rangle$ is left fixed by $\rho\left(\pi^{t}\right)$ if and only if $t$ is a multiple of $p_{r}$, i.e., $t=m p_{r}$ for some integer $m$. Therefore, the number of the pairs $\left\langle u_{r s}, Q_{r}\right\rangle$ is clearly $p_{r}\left(\begin{array}{c}p_{r}-1 \\ q_{r}\end{array}\right)$.

On the other hand, the arc $\left\langle u_{r s}, Q\right\rangle$ is left fixed by $\rho\left(\pi^{t}\right)$ if and only if $\left\langle u_{r s}, Q_{r}\right\rangle$ and $Q_{i}$ are left fixed by $\rho\left(\pi^{t}\right)$ for each $i \in\{1,2, \cdots, k\} \backslash\{r\}$. In this case we have $t=m p_{r}$ for some integer $m$. Therefore, the number of such arcs is equal to

$$
\Psi\left(\rho^{t}(P)\right)=p_{r}\left(\begin{array}{c}
p_{r}-1 \\
q_{r}
\end{array}\right) \prod_{i \neq r}\left(\begin{array}{l}
\eta_{i m} \\
\xi_{i m}
\end{array}\right)
$$

if $p_{i} / \eta_{i m}$ divides $q_{i}$ for each $i \in\{1,2, \cdots, k\} \backslash\{r\}$ and is 0 for otherwise, where $\eta_{i m}=$ $\left(m p_{r}, p_{i}\right), \xi_{i m}=q_{i} \eta_{i m} / p_{i}$.

Let $\operatorname{lcm}\left(\mathcal{Q}_{r}\right)$ be the least common multiple of $p_{r}$ and those $p_{i}$ with $q_{i} \neq 0, i=$ $1,2, \cdots, k$. Then,

$$
\begin{aligned}
\Omega(\rho(P)) & =\frac{1}{\left[p_{1}, p_{2}, \cdots, p_{k}\right]} \sum_{t=1}^{\left[p_{1}, p_{2}, \cdots, p_{k}\right]} \Psi\left(\rho^{t}(P)\right) \\
& =\frac{1}{\left[p_{1}, p_{2}, \cdots, p_{k}\right]} \sum_{\mathcal{Q}} \sum_{r=1}^{k} \frac{\left[p_{1}, p_{2}, \cdots, p_{k}\right]}{\operatorname{lcm}\left(\mathcal{Q}_{r}\right)} \sum_{m \in M_{r}} \Psi\left(\rho^{t}(P)\right),
\end{aligned}
$$

where $\eta_{i m}=\left(m p_{r}, p_{i}\right), \xi_{i m}=q_{i} \eta_{i m} / p_{i}$ and

$$
M_{r}=\left\{m \in\left\{1,2, \cdots, \operatorname{lcm}\left(\mathcal{Q}_{r}\right) / p_{r}\right\}: p_{i} / \eta_{i m} \text { divides } q_{i}, i=1,2, \cdots, k ; i \neq r\right\} .
$$

Combining with (2)-(5), we reach the following result. 
Theorem 5. The number of unlabeled q-uniform directed hypergraphs of order $n$ is given by

$$
d_{q}(n)=\sum_{P \in \mathcal{P}(n)} \frac{1}{1^{\alpha_{1}} 2^{\alpha_{2}} \cdots n^{\alpha_{n}} \alpha_{1} ! \alpha_{2} ! \cdots \alpha_{n} !} 2^{\Omega(\rho(P))},
$$

where $\Omega(\rho(P))$ is defined as in (5).

In the following we give the more concise expressions of $\Omega_{q}(P)$ for $q \in\{2,3\}$, in terms of $p_{i}, i=1,2, \cdots, k$. The result for $q=2$ (Corollary 6 ), i.e., the ordinary directed graphs, simplifies the one given by Harary and Palmer [9] by using the generating function approach.

Corollary 6.

$$
\Omega_{2}(P)=n-k+\sum_{i<j} 2\left(p_{i}, p_{j}\right) .
$$

Proof. For convenience, we write

$$
\omega\left(\mathcal{Q}_{r}\right)=\frac{1}{\operatorname{lcm}\left(\mathcal{Q}_{r}\right)} \sum_{m \in M_{r}} p_{r}\left(\begin{array}{c}
p_{r}-1 \\
q_{r}
\end{array}\right) \prod_{i \neq r}\left(\begin{array}{l}
\eta_{i m} \\
\xi_{i m}
\end{array}\right) .
$$

Since $q=2$, we have two cases to discuss.

Case 1. $q_{i}=1$ for some $i \neq r$ and $q_{j}=0$ for all $j \neq i$.

In this case, we have $\operatorname{lcm}\left(\mathcal{Q}_{r}\right)=\left[p_{i}, p_{r}\right]$. Let $m \in M_{r}$. Since $q_{i}=1$, then $p_{i} /\left(m p_{r}, p_{i}\right) \mid$ $q_{i}$ (i.e., $p_{i} /\left(m p_{r}, p_{i}\right)$ divides $\left.q_{i}\right)$ implies that $p_{i} \mid m p_{r}$. Therefore, $\left[p_{i}, p_{r}\right] \mid m p_{r}$. On the other hand, by the definition of $M_{r}, m \leqslant \operatorname{lcm}\left(\mathcal{Q}_{r}\right) / p_{r}=\left[p_{i}, p_{r}\right] / p_{r}$. So $m=\left[p_{i}, p_{r}\right] / p_{r}$, i.e., $M_{r}=\left\{\left[p_{i}, p_{r}\right] / p_{r}\right\}$. Noticing that $q_{r}=0$, we have

$$
\omega\left(\mathcal{Q}_{r}\right)=\frac{1}{\left[p_{i}, p_{r}\right]} p_{r}\left(\begin{array}{c}
p_{r}-1 \\
q_{r}
\end{array}\right)\left(\begin{array}{c}
p_{i} \\
1
\end{array}\right)=\left(p_{i}, p_{r}\right) .
$$

The sum over all the $i$ 's and the root subscript $r$ 's with $r \neq i$ is clearly

$$
\sum_{i<j} 2\left(p_{i}, p_{j}\right)
$$

Case 2. $q_{r}=1$ and $q_{i}=0$ for all $i \neq r$.

In this case we have $\operatorname{lcm}\left(\mathcal{Q}_{r}\right)=p_{r}$ and $\omega\left(\mathcal{Q}_{r}\right)=p_{r}-1$ directly. The sum over all $r$ 's is clearly $\sum_{r=1}^{k}\left(p_{r}-1\right)=n-k$.

\section{Corollary 7.}

$$
\begin{aligned}
\Omega_{3}(P)= & \sum_{i=1}^{k}\left(\begin{array}{c}
p_{i}-1 \\
2
\end{array}\right)+3 \sum_{i<j<h}\left(p_{i}, p_{j}, p_{h}\right)^{2} \\
& +\frac{1}{4} \sum_{i<j}\left(p_{i}, p_{j}\right)\left(6 p_{i}+6 p_{j}-12+\left|(-1)^{\left[p_{i}, p_{j}\right] / p_{i}}-(-1)^{\left[p_{i}, p_{j}\right] / p_{j}}\right|\right) .
\end{aligned}
$$


Proof. Since $q=3$, we have four cases to discuss.

Case 1. $q_{i}=q_{j}=1(i, j \neq r)$ and $q_{h}=0$ for all $h \neq i, j$.

The discussion for this case is similar to Case 1 in the proof of Corollary 6 , which yields

$$
\omega\left(\mathcal{Q}_{r}\right)=\frac{1}{\left[p_{i}, p_{j}, p_{r}\right]} p_{r}\left(\begin{array}{c}
p_{r}-1 \\
q_{r}
\end{array}\right)\left(\begin{array}{c}
p_{i} \\
1
\end{array}\right)\left(\begin{array}{c}
p_{j} \\
1
\end{array}\right)=\left(p_{i}, p_{j}, p_{r}\right)^{2} .
$$

The sum over all the triples $i, j, r$ with root subscript $r$ is clearly

$$
3 \sum_{i<j<h}\left(p_{i}, p_{j}, p_{h}\right)^{2} .
$$

Case 2. $q_{i}=q_{r}=1$ and $q_{j}=0$ for all $j \neq i, r$.

The discussion is similar to that in Case 1 which yields that

$$
\omega\left(\mathcal{Q}_{r}\right)=\frac{1}{\left[p_{i}, p_{r}\right]} p_{r}\left(\begin{array}{c}
p_{r}-1 \\
q_{r}
\end{array}\right)\left(\begin{array}{c}
p_{i} \\
1
\end{array}\right)=\left(p_{r}-1\right)\left(p_{i}, p_{r}\right) .
$$

The sum over all the pairs $r$ and $i$ with root subscript $r$ is clearly

$$
\sum_{i<j}\left(p_{i}+p_{j}-2\right)\left(p_{i}, p_{j}\right)
$$

Case 3. $q_{i}=2, i \neq r$, and $q_{j}=0$ for all $j \neq i$.

In this case, $\operatorname{lcm}\left(\mathcal{Q}_{r}\right)=\left[p_{i}, p_{r}\right]$. Suppose $m \in M_{r}$. Then we have $p_{i} /\left(m p_{r}, p_{i}\right)=1$ or $p_{i} /\left(m p_{r}, p_{i}\right)=2$.

If $p_{i} /\left(m p_{r}, p_{i}\right)=1$ then $m p_{r}=\left[p_{i}, p_{r}\right]$ since $m \leqslant\left[p_{i}, p_{r}\right] / p_{r}$.

If $p_{i} /\left(m p_{r}, p_{i}\right)=2$ then $m p_{r}=\alpha p_{i} / 2$, where $\alpha$ is an odd integer. Notice that the equation $2 m p_{r}=\alpha p_{i}$ implies that $\left[p_{i}, p_{r}\right] \mid \alpha p_{i}$ and $\left[p_{i}, p_{j}\right] \mid 2 m p_{r}$, i.e., $\alpha p_{i}=2 m p_{r}=$ $l\left[p_{i}, p_{r}\right]$ for some integer $l \geqslant 1$. On the other hand, we note that $m p_{r}=\alpha p_{i} / 2 \neq\left[p_{i}, p_{r}\right]$ since $\alpha$ is odd. Therefore, $m p_{r}=\alpha p_{i} / 2<\left[p_{i}, p_{r}\right]$ since $m \leqslant \operatorname{lcm}\left(\mathcal{Q}_{r}\right) / p_{r}=\left[p_{i}, p_{r}\right] / p_{r}$. This means that $\alpha p_{i}=2 m p_{r}=\left[p_{i}, p_{r}\right]$. Thus, $m=\left[p_{i}, p_{j}\right] / 2 p_{r}$, which is valid only when $\left[p_{i}, p_{r}\right] / p_{i}$ is odd and $\left[p_{i}, p_{r}\right] / p_{r}$ is even.

The above discussion shows that $M_{r}=\left\{\left[p_{i}, p_{r}\right],\left[p_{i}, p_{r}\right] / 2\right\}$ if $\left[p_{i}, p_{r}\right] / p_{i}$ is odd and $\left[p_{i}, p_{r}\right] / p_{r}$ is even, or $M_{r}=\left\{\left[p_{i}, p_{r}\right]\right\}$ for otherwise. Thus,

$$
\omega\left(\mathcal{Q}_{r}\right)=\frac{1}{\left[p_{i}, p_{r}\right]} p_{r}\left(\begin{array}{c}
p_{r} \\
0
\end{array}\right)\left(\left(\begin{array}{c}
p_{i} \\
2
\end{array}\right)+\left(\begin{array}{c}
p_{i} / 2 \\
1
\end{array}\right)\right)=\left(p_{i}, p_{r}\right)\left(\frac{p_{i}-1}{2}+\frac{1}{2}\right)
$$

if $\left[p_{i}, p_{r}\right] / p_{i}$ is odd and $\left[p_{i}, p_{r}\right] / p_{r}$ is even, or

$$
\omega\left(\mathcal{Q}_{r}\right)=\left(p_{i}, p_{r}\right) \frac{p_{i}-1}{2}
$$

for otherwise. We note that the same discussion can be also taken for the case when $i$ represents the root subscript and $q_{r}=2$. We denote it by $\mathcal{Q}_{i}$. Combining these two cases, if $\left[p_{i}, p_{r}\right] / p_{i}$ is odd and $\left[p_{i}, p_{r}\right] / p_{r}$ is even or $\left[p_{i}, p_{r}\right] / p_{r}$ is odd and $\left[p_{i}, p_{r}\right] / p_{i}$ is even, then

$$
\omega\left(\mathcal{Q}_{r}\right)+\omega\left(\mathcal{Q}_{i}\right)=\frac{1}{2}\left(p_{i}, p_{r}\right)\left(p_{i}+p_{r}-2+1\right) .
$$


Table 2: Numerical results for $d_{2}(n)$ and $d_{3}(n)$ with $n=1,2, \cdots, 13$.

\begin{tabular}{lll}
\hline$n$ & $d_{2}(n)$ & $d_{3}(n)$ \\
\hline 1 & 1 & 1 \\
2 & 3 & 1 \\
3 & 16 & 4 \\
4 & 218 & 218 \\
5 & 9608 & 8978144 \\
6 & 1540944 & 16012858855249024 \\
7 & 882033440 & 8048575244466823237217930240 \\
8 & 1793359192848 & $92793754751169593095822191027513 \times 10^{14}$ \\
9 & 13027956824399552 & $19943247292031145872447822753972 \times 10^{39}$ \\
10 & 341260431952972580352 & $64719537664622829251804470296850 \times 10^{70}$ \\
11 & 32522909385055886111197440 & $25626667592781594075154365586886 \times 10^{110}$ \\
12 & 11366745430825400574433894004222 & $99875777723160235238919560135473 \times 10^{158}$ \\
13 & $14669085692712929869037096075314 \times 10^{6}$ & $30864266716019372520365057557707 \times 10^{217}$ \\
\hline
\end{tabular}

Otherwise, we have

$$
\omega\left(\mathcal{Q}_{r}\right)+\omega\left(\mathcal{Q}_{i}\right)=\frac{1}{2}\left(p_{i}, p_{r}\right)\left(p_{i}+p_{r}-2\right) .
$$

Both of the above two equations could be written as the following form:

$$
\omega\left(\mathcal{Q}_{r}\right)+\omega\left(\mathcal{Q}_{i}\right)=\frac{1}{4}\left(p_{i}, p_{r}\right)\left(2 p_{i}+2 p_{r}-4+\left|(-1)^{\left[p_{i}, p_{r}\right] / p_{i}}-(-1)^{\left[p_{i}, p_{r}\right] / p_{r}}\right|\right) .
$$

Case 4. $q_{r}=2$ and $q_{i}=0$ for all $i \neq r$.

In this case we have $\operatorname{lcm}\left(\mathcal{Q}_{r}\right)=p_{r}$ and

$$
\omega\left(\mathcal{Q}_{r}\right)=\frac{1}{\operatorname{lcm}\left(\mathcal{Q}_{r}\right)} p_{r}\left(\begin{array}{c}
p_{r}-1 \\
q_{r}
\end{array}\right)=\left(\begin{array}{c}
p_{r}-1 \\
2
\end{array}\right) .
$$

The corollary now follows directly by combining (6)-(9).

By Corollary 6 and Corollary 7, the numerical results of the number of unlabeled $q$-uniform directed hypergraphs with $q=2,3$ and the number of vertices from 1 up to 13

are listed in Table 2, as an example. The results with the number of vertices up to 8 for 2-uniform directed hypergraphs (i.e., the ordinary directed graphs) were also listed in [9].

\section{References}

[1] G. Ausiello, G.F. Italiano, L. Laura, U. Nanni, F. Sarracco. Structure Theorems for Optimum Hyperpaths in Directed Hypergraphs, In ISCO 2012, LNCS 7422, A.R. Mahjoub et al. (Eds.), Springer-Verlag, Berlin Heidelberg, 2012, pp. 1-14.

[2] E.F. Beckenbach. Applied Combinatorial Mathematics. Wiley, New York, 1964.

[3] Vivek S. Borkar, V. Ejov, Jerzy A. Filar and Giang T. Nguyen. Graph Enumeration, Hamiltonian Cycle Problem and Markov Chains. In International Series in Operations Research \& Management Science, 171(Part 5):179-192, 2012. 
[4] N. G. de Bruijn and D. A. Klarner. Enumeration of generalized graphs. Indag. Math., 31:1-9, 1969.

[5] G. Chapuy, E. Fusy, O. Giménez, B. Mohar and M. Noy. Asymptotic enumeration and limit laws for graphs of fixed genus. J. Combin. Theory, Ser. A, 118:748-777, 2011.

[6] A. Frank, T. Király and Z. Király. On the orientationof graphs and hypergraphs. Discrete Appl. Math., 131:385-400, 2003.

[7] O. Giménez. Asymptotic enumeration and limit laws of planar graphs, J. Amer Math. Soc., 22:309-329, 2009.

[8] O. Giménez and M. Noy, Counting planar graphs and related families of graphs. Surveys in Combinatorics 2009, Cambridge University Press, Cambridge, 2009, pp. 169-210.

[9] F. Harary and E. M. Palmer. Graphical Enumeration. Academic Press, New York, 1973.

[10] F. Harary. On the number of bi-colored graphs. Pac. J. Math., 8:743-755, 1958.

[11] F. Harary, E.M. Palmer, R.W. Robinson and A.J. Schwenk. Enumeration of Graphs with Signed Points and Lines. J. Graph Theory, 1:295-308, 1977.

[12] T. Ishihara. Enumeration of Hypergraphs. Europ. J. Combin., 22:503-509, 2001.

[13] K. Li and L.S. Wang. A polynomial time approximation scheme for embedding a directed hypergraph on a ring. Information Processing Letters, 97:203-C207, 2006.

[14] E.M. Palmer, R.C. Read and R.W. Robinson. Counting claw-free cubic graphs. SIAM J. Discrete Math., 16:65-73, 2003.

[15] K. R. Parthasarathy. Enumeration of graphs with given partition. Canad. J. Math., 20:40-47, 1968.

[16] G. Pólya and R.C. Read. Combinatorial Enumeration of Groups, Graphs, and Chemical Compounds. Springer, Berlin, 1987.

[17] R. Robinso. Counting cubic graphs. J. Graph Theory, 1:285-286, 1977.

[18] I. T. Skibenko. Enumeration of hypergraphs. I. Cybernetics and Systems Analysis, 20:167-172, 1984.

[19] S. Tazawa and T. Shirakura. Enumeration of unlabelled bicolored graphs by degree parities, J. Stat. Plan. Infer., 58:193-206, 1977.

[20] A. Vietri. The complexity of arc-colorings for directed hypergraphs. Discrete Appl. Math., 143:266-271, 2004.

[21] S. Y. Wu. Enumeration of hypergraphs. Soochow J. Math., 6:167-175, 1980. 